\title{
Mechanical and Electrical Properties: Electrospun Alginate/Carbon Nanotube Composite Nanofiber
}

\author{
Md. Shahidul Islam", Md. Ashaduzzaman', Shah Md. Masum and Jeong Huyn Yeum² \\ ${ }^{1}$ Department of Applied Chemistry and Chemical Engineering, University of Dhaka, Dhaka-1000, Bangladesh \\ ${ }^{2}$ Department of Advanced Organic Materials Science and Engineering, Kyungpook National University, Daegu 702-701, \\ South Korea.
}

Received on 10.08.2011. Accepted for Publication on 08. 10. 2011

\begin{abstract}
Nanofibers of the composite of alginate (Alg) and carbon nanotube (CNT) were prepared using electrospinning method out of aqueous solutions. Scanning electron microscopy (SEM), transmission electron microscopy (TEM), mechanical measurement, and electrical conductivity were done to characterize the Alg/CNT composite nanofibers morphology and properties. The SEM and TEM images show the CNT to be well incorporated along the nanofibers. The study shows that the introduction of CNT results in improvement in tensile strength and electrical conductivity of the Alg matrix. The electrospinning of Alg/CNT composites could potentially supply useful options for the fabrication of biomaterial scaffolds.
\end{abstract}

Keywords: Alginate, Carbon nanotube, Electrospinning, Nanofiber.

\section{Introduction}

The electrospinning technique has attracted great interest among academic and industrial scientists because it is very simple and effective approach to produce nanofibers, which have been found to be attractive for various applications in biomedical engineering, filtration, protective clothing, catalysis reactions, and sensors ${ }^{1-5}$. Doshi and Reneker ${ }^{6}$ reported the main principle of the electrospinning technique.

Alginate employed in this work is an abundant polysaccharide, which can be supplied in plenty from marine algae. It is well known that alginate is a copolymer composed of $\beta$-D-mannuronate (M) and $\alpha$-L-guluronate (G) resides in various $\mathrm{M} / \mathrm{G}$ ratios; these resides are arranged in a block-wise fashion, constructed not only of homopolymer blocks (MM or GG) but also alternating blocks $(\mathrm{MG})^{7-8}$. This carbohydrate polymer has been re-evaluated recently as an attractive natural resource, possessing a potential to be further developed for medical, pharmaceutical, and bio- and other industrial applications. In contrast to the numerous studies carried out on the physical properties and industrial applications of alginate gels ${ }^{9-14}$.

Carbon nanotubes were first reported by Iijima in 1991 and they possess unique mechanical, opt ical, electrical and thermal conductivity along with thermal and chemical stability $^{15-17}$. Due to the unique properties of CNT, researches have focused on utilizing these remarkable characteristics for engineering applications such as polymeric composites, hydrogen storage ${ }^{18}$, actuators ${ }^{19}$, chemical sensors ${ }^{20}$, and nanoelectronic devices ${ }^{21}$. Carbon nanotubes have enormous high surface-to-volume ratio, and that's why they offer chemically facile sites that can be functionalized with additives thereby resulting in a strong interfacial bond with the matrix.

Research on Alg/CNT composite nanofibers by electrospinning technique has not been a focus in enormously. To the best of our knowledge, no reports are available on the morphology of $\mathrm{Alg} / \mathrm{CNT}$ composite nanofibers applying electrospinning technique. In this report, $\mathrm{Alg} / \mathrm{CNT}$ composite nanofibers are fabricated by using the electrospinning technique. Moreover, mechanical and electrical properties are exhibited of this type of composite nanofibers.

\section{Experimental}

$4 \mathrm{wt} \%$ alginate solution was first prepared by dissolving alginate powder in deionized water, followed by centrifugation to remove air bubbles. Then CNT dispersion was mixed in that solution under magnetic stirring for $1 \mathrm{~h}$ at room temperature. Solution containing $0-1.0 \mathrm{wt} \%$ of Triton $\mathrm{X}-100^{\mathrm{TM}}$ and $0-10 \mathrm{wt} \%$ of dimethyl formamide (DMF) was mixed with the alginate solution, and the mixture was stirred for additional $3 \mathrm{~h}$ and centrifuged to remove air bubbles. Solution viscosity in the electrospinnability was accomplished by maintaining the alginate solution at room temperature over a period of 25 days and measuring the viscosity of the solution at different time points. During electrospinning, high-voltage power was applied to the $\mathrm{Alg} / \mathrm{CNT}$ solution in a syringe via an alligator clip attached to the syringe needle. The applied voltage was adjusted at $15 \mathrm{kV}$. The solution was delivered to the blunt needle tip via a syringe pump to control the solution flow rate. Fibers were collected on electrically grounded aluminum foil placed at a 
$15 \mathrm{~cm}$ vertical distance to the needle tip. The morphology and property characterization of the electrospun Alg/CNT composite nanofibers were conducted with scanning electron microscope (model JSM-6380, JEOL) after gold coating, and transmission electron microscopy (TEM) (HITACHI, model H-7600) with an accelerating voltage of $100 \mathrm{kV}$. The viscosities of the alginate solution was measured using a Haake Viscometer (VT550) equipped with double concentric cylinder-type SP2P sensors. Tensile strength was carried out by the ZWICK Z005 (ZWICK materials testing machine, Germany). Conductivity was measured by solartron SI 1287 electrochemical station.

\section{Results and Discussion}

The high viscosity of a pure alginate solution is believed primarily due to strong intermolecular interaction between polymer chains and possible entanglements which act as knots (i.e. the physical junctions of alginate gels). The decrease in viscosity by storing the alginate solution can be attributed to the changes in inter- and intra-molecular interactions of alginate chains. As shown in Fig 1, the viscosity of the solution dropped considerably in the first two weeks and gradually saturated thereafter. The viscosity drop with the storage time was believed attributable to a decrease in the average molecular weight of the alginate as a result of polymer chain degradation. It was assumed that alginate molecular chain scission was caused by the breakage of glycosidic bonds due to aqueous hydrolysis ${ }^{22}$.

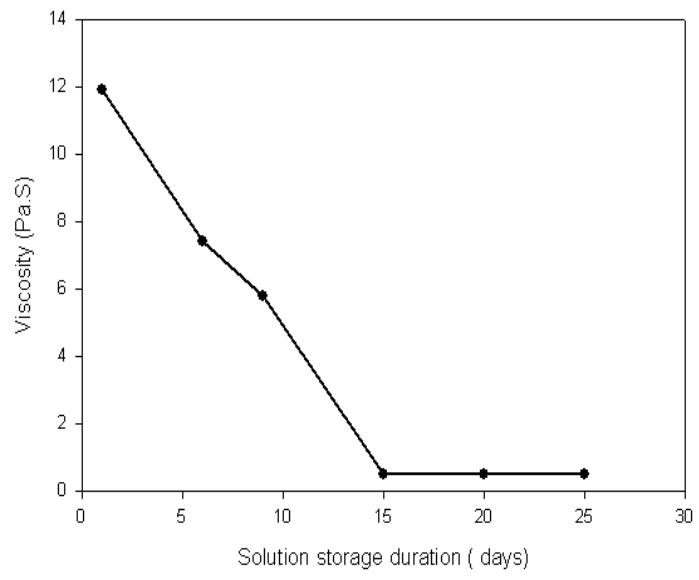

Fig.1. Viscosity of Alginate solution as a function of storage time.

Fig 2 demonstrates SEM images of nanofibers of Alg/CNT composite obtained from 4 wt.\% Alg solution with various concentrations of $\mathrm{CNT}(0,0.1,0.5$, and $1 \mathrm{wt} . \%)$. It is clearly seen that 0.1 , and 0.5 wt.\% CNT content do not effects enormously on the morphology of electrospun Alg nanofibers (Figs.2b \& 2c), where as, $1 \mathrm{wt} \% \mathrm{CNT}$ content shows the effect to a some extent on the formation of electrospun Alg nanofibers (Fig.2d).
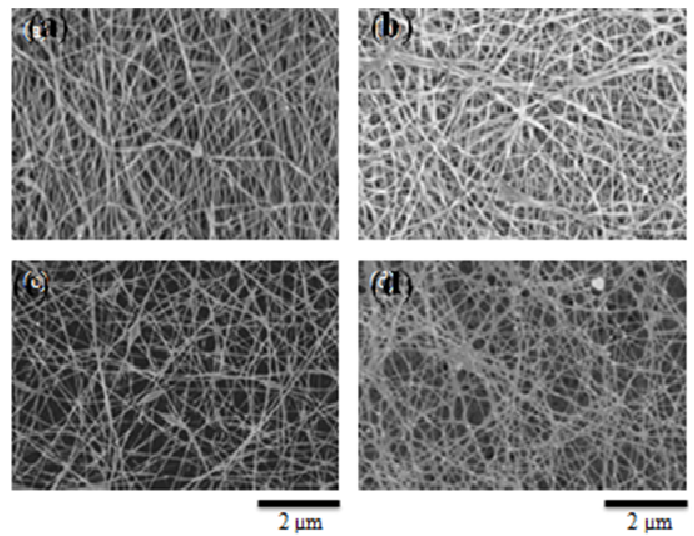

Fig.2. SEM images of electrospun Alginate/CNT composite nanofiber prepared using different CNT contents of (a) 0, (b) 0.1, (c) 0.5 , and (d) $1 \mathrm{wt} . \%$ ( Total polymer concentration $=4 \mathrm{wt} . \%$, Solution storage time $=15$ days, Applied voltage $=15 \mathrm{kV}$, and $\mathrm{TCD}=15 \mathrm{~cm})$.

Fig 3 shows the TEM images of electrospun Alg/CNT hybrid nanofibers containing $0,0.1,0.5$ and 1 wt. $\%$ of CNT. It is evident that the quantity of CNT content increases in that hybrid nanofibers with augmenting the wt.\% of CNT and it is also plain and clear that the CNT is well embedded and aligned along the fiber axis.
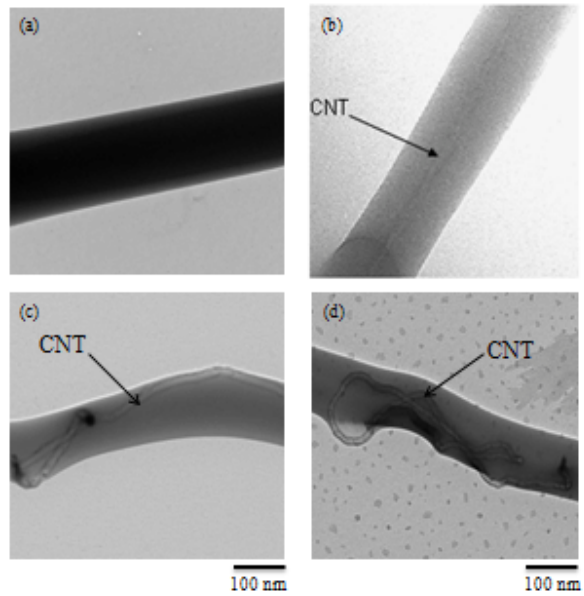

Fig. 3. TEM images of electrospun Alginate/CNT composite nanofiber prepared using different CNT contents of (a) 0 , (b) 0.1 , (c) 0.5 , and (b) 1 wt.\% (Total polymer concentration $=4$ wt. $\%$, Solution storage time $=15$ days, Applied voltage $=15 \mathrm{k} \mathrm{V}$, and $\mathrm{TCD}=15 \mathrm{~cm}$ ).

\section{Tensile strength}

Fig 4 illustrates the tensile strength in dependence on the filler content. It can be found that the carbon nanotubes can improve the tensile strength of polymer matrix obviously. With augmenting the concentration of carbon nanotubes, the 
tensile strength of the composite nanofibers increases. However, when the addition of CNT is over $0.5 \mathrm{wt} . \%$, the extra CNT putting together will cause a decrease of the mechanical properties. In this study, $0.5 \mathrm{wt} . \%$ carbon nanotube addition has the best effect on the mechanical properties of the matrix. . The mainly probable reason is that a carbon nanotubes network structure is formed, which can take more mechanical loading from the matrix when the matrix is under stress. This means that when the applied loading is over the elastic deformation stress, the carbon nanotubes have a stress transfer effect, ${ }^{23-24}$ which can enhance the strength and plasticity of the Alg matrix. When the content of carbon nanotubes is less than $0.5 \%$, the carbon nanotubes are dispersed uniformly in the matrix, without obvious agglomerating. However, too much CNT (more than 0.5 wt.\%) will do harm to the matrix, and the strength of composite nanofibers will be lower than that of 0.5 wt.\% CNT. This may be resulted from the bad dispersion of too much more CNT. The extra CNT will aggregate more seriously due to their high aspect ratio, and produce a lot of defects in the matrix and degrade the mechanical properties to a some extent.

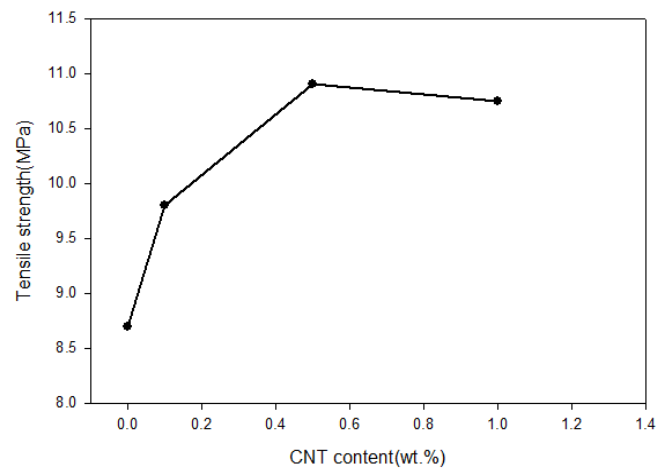

Fig.4. Tensile strength of electrospun Alginate/CNT composite nanofiber with various CNT contents ( Total polymer concentration $=4$ wt. $\%$, Solution storage time $=15$ days, Applied voltage $=15 \mathrm{k} \mathrm{V}$, and TCD $=15 \mathrm{~cm}$ ).

\section{Electrical Conductivity}

The resistivity of Alg/CNT composite nanofibers with different contents of carbon nanotubes was measured by solartron SI 1287 electrochemical station. Fig 5 shows the logarithm relationship between the volume resistance and the content of carbon nanotubes. It can be found that with increasing addition of carbon nanotubes, the resistivity of the composite decreases dramatically. This means the conductivity of the composite nanofibers increase greatly. According to the percolation theory, when the content of carbon nanotubes is low, the carbon nanotubes distribute randomly in the matrix, and do not form a conductive network. At this stage, the conductivity of composite nanofibers mainly depends on the conductivity of the matrix. With increasing the content of carbon nanotubes, they begin to connect and interact with each other to form a network. The above percolation threshold values, the continuous conductive channel is developed in the frame of the network, the resistivity will decrease greatly. The percolation threshold values are found at $0.21 \mathrm{wt} . \%$ and 0.5 wt.\% CNT.

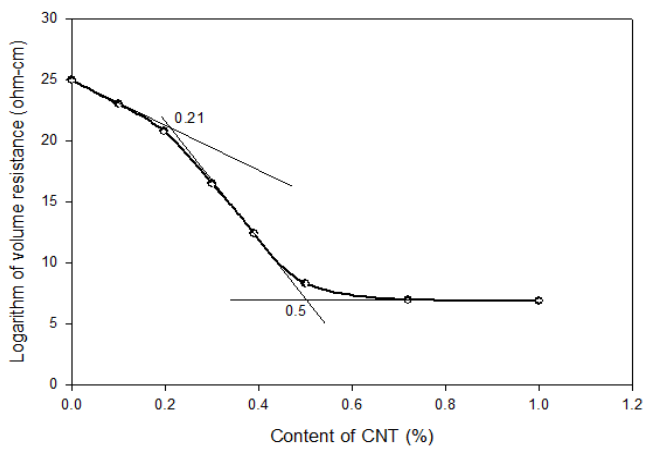

Fig. 5. Effect of CNT on volume resistance.

\section{Conclusion}

$\mathrm{Alg} / \mathrm{CNT}$ composite nanofiber could be fabricated by the electrospinning method in aqueous solutions. It is seen that the incorporation of CNT results in improvement in tensile strength and electrical conductivity of the Alg matrix. Coexistence and alignment of CNT in alginate nanofiber is found by TEM micrographs.

1. Reneker, D. H., I. Chun, 1996. Nanometer diameter fibers of polymer, produced by Nanotechnology. 7, 216-223.

electrospinning.

2. Zussman, E., A. Theron, A. L. Yarin, 2003. Formation of nanofiber crossbars in electrospinning. Appl. Phys. Lett. 82, 973-975.

3. Li, D., Y. Xia, 2004. Electrospinning of nanofibers: Reinventing the whell. Adv. Mater. 16,1151-1170.

4. Han, X. J., Z. M. Huang, C. L. He, L. Liu, Q. S. Wu, 2006. Coaxial electrospinning of PC (shell)/PU(core) composite nanofibers for textile application. Polym. Compos. 27, 381387.

5. Cui, W., Li, X., S. Zhou, J. J. Weng, 2007. Investigation on process parameters of electrospinning systems through orthogonal experimental design. Appl. Polym. Sci. 103, 31053112 .

6. Doshi, J., D. H. Reneker, 1995. Electrospinning process and applications of electrospun fibers. J. Electrostat. 35, 151-160.

7. Fischer, F. G., H. Dorfel, 1955. The polyuronic acids of brown algae. Hoppe Seyler's Z . Physiol. Chem. 302, 186203.

8. Huang, A., B. Larsen, O. Smidsrod, 1966. A study of the constitution of alginic acid by partial acid hydrolysis. Acta. Chem. Scand. 20, 183-190.

9. Cottrell, I. W., P. Kovacs, 1980. Handbook of Water-Soluble Gums and Resins: Alginates. McGraw-Hill: New York. p 214. 
10. Whistler, R. L., J. N. Bemiller, 1993. Industrial Gums Polysaccharides and Their Derivatives; Academic Press: San Diego.

11. Glicksman, M., 1969. Food Science and Technology, Gum Technology in the Food Industry. Academic Press, New York. p239.

12. Imeson, A., 1997. Thickening and Gelling Agent for Food. Chapter 2: Alginates, Blackie: London, New York. p23.

13. Kuo, C. K., P. X. Ma, 2001. Ionically crosslinked alginate hydrogels as scaffolds for tissue engineering: part 1 . Structure, gelation rate and mechanical properties. Biomaterials. 22, 511-521.

14. Balakrishnan, B., M. Mohanty, P. R. Umashankar, Jayakrishnan, A., 2005. Evaluation of an in situ forming hydrogel wound dressing based on oxidized alginate and gelatin. Biomaterials. 26, 6335-6342.

15. Iijima, S., 1991. Helical microtubules of graphitic carbon. Nature. 354, 56-58.

16. Wong, E. W., P. E. Sheehan, C. M. Lieber, 1997. Nanobeam mechanics: elasticity, strength, and toughness of nanorods and nanotubes. Science. 277, 1971-1975.

17. Yang, J., Y. Lin, J. Wang, M. Lai, J. Li, J. Liu, X. Tong, H. Cheng, 2005. Morphology, thermal stability, and dynamic mechanical properties of atactic polypropylene/carbon nanotube composites. Appl. Polym. Sci. 98,1087-1091.
18. Liu, C., Y. Y. Fan, M. Liu, H. T. Cong, H. M. Cheng, M. S. Dresselhaus, 1999. Hydrogen storage in single-walled carbon nanotubes at room temperature. Science. 286, 1127-1129.

19. Baughman, R. H., C. Cui, A. A. Zakhidov, Z. Iqbal, J. N. Barisci, G. M. Spinks, et al., 1999. Carbon nanotube actuators. Science. 284, 1340-1344.

20. Kong, J., N. R. Franklin, C. Zhou, M. G. Chapline, S. Peng, K. Cho, et al., 2000. Nanotube molecular wires as chemical sensors. Science. 287, 622-625.

21. Tans, S. J., A. R. M. Verschueren, C. Dekker, 1998. Roomtemperature transistor based on a single carbon nanotube. Nature. 393, 49-52.

22. Wasikiewicz, J. M., F. Yoshii, N. Nagasawa, R. A. Wach, H. Mitomo, 2005. Degradation of chitosan and sodium alginate by gamma radiation, sonochemical and ultraviolet methods. Radiat. Phys. Chem. 73, 287-295.

23. Zhang, P., P. E. Lammert, V. H. Crespi, 1998. Plastic Deformation of Carbon Nnaotubes. Phys. Rev. Lett. 81, 53465349.

24. Richard, P., T. Prasse, J. Y. Cavaille, L. Chazeau, C. Gauthier, J. Duchet, 2003. Reinforcement of rubbery epoxy by carbon nanofibers. Mater. Sci. Eng. A. 352, 344-348. 